\title{
A hybrid approach of template synthesis and electroless depositing for Ni-W-P nanoparticles
}

\author{
Jianhua Zhou • Jianping He • Tao Wang • Xiu Chen
}

Received: 1 July 2008 /Revised: 28 August 2008 / Accepted: 8 September 2008

(C) Springer-Verlag 2008

This article has been retracted by the journal as it has been published elsewhere.

This article has been retracted by the journal as it has been published elsewhere.

J. Zhou $\cdot$ J. He $(\bowtie) \cdot$ T. Wang $\cdot$ X. Chen College of Material Science and Technology,

Nanjing University of Aeronautics and Astronautics,

Nanjing 210016, People's Republic of China

e-mail: jianph@nuaa.edu.cn 